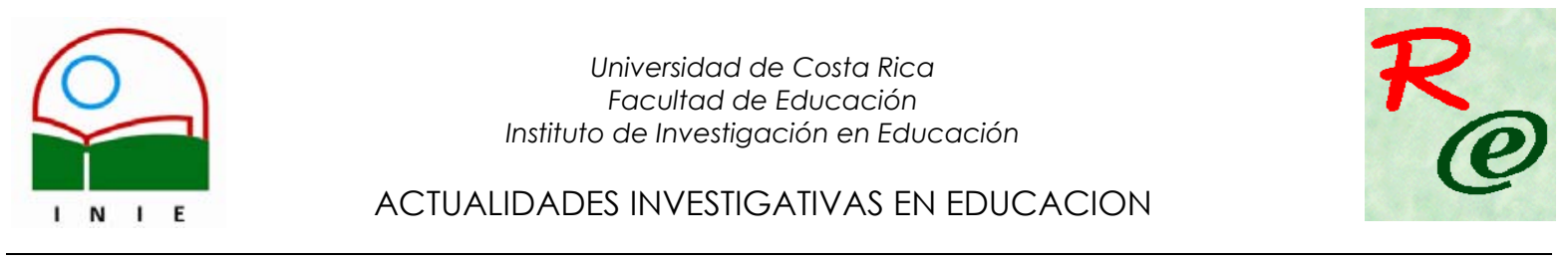

\title{
PENSUM* NO LINEAL: UNA PROPUESTA INNOVADORA PARA EL DISEÑO DE PLANES DE ESTUDIO
}

\author{
Alberto J. Cañas ${ }^{1}$ \\ Eleonora Badilla Saxe ${ }^{2}$
}

Resumen: El propósito de este artículo, es presentar organizadores conceptuales (como el pensamiento complejo, el aprendizaje significativo y los mapas conceptuales) que, con la ayuda de organizadores tecnológicos multimediales (como Internet, la WWW y el CmapTools), permiten el diseño de pensum (programas o planes de estudio) no lineales, que evidencien la muti-relacionalidad de sus partes, como una alternativa para la educación en el mundo actual.

Palabras clave: PENSUM/ PLAN DE ESTUDIOS/ PROGRAMA DE ESTUDIOS/ NO LINEAL/ DISEÑO CURRICULAR/ TECNOLOGÍA MULTIMEDIAL/ MAPAS CONCEPTUALES/ PENSAMIENTO COMPLEJO/ APRENDIZAJE SIGNIFICATIVO/ EDUCACIÓN PRESENCIAL/ EDUCACIÓN BIMODAL/ EDUCACIÓN VIRTUAL/

\begin{abstract}
The purpose of this article is to present conceptual (such as Complex Thinking, Meaningful Learning and Concept Mapping) and technological (such as Internet, the WWW and CmapTools) organizers that allow a non-linear pensum design that can make visible its multiple interrelations.
\end{abstract}

Keywords: PENSUM/ CURRICULUM/ NON-LINEAR/ MULTIMEDIA TECHNOLOGY/ CONCEPT MAPS/ COMPLEX THINKING/ MEANINGFUL LEARNING/VIRTUAL LEARNING/ E-LEARNING/ EDUCATION/

\begin{abstract}
${ }^{1}$ Doctorado en Ciencias de la Gestión por la Universidad de Waterloo, Canadá. Es subdirector del Institute for Human and Machine Cognition (IHMC) en Pensacola, Florida; es consultor principal del proyecto Conéctate al Conocimiento de Panamá y es miembro fundador de la Academia Internacional de Tecnología y Conocimiento AiCyT.

${ }^{2}$ Maestría en Tecnologías digitales en educación de la Universidad de Hartford, Connecticut. Es profesora catedrática de la Universidad de Costa Rica donde dirige el Centro de Evaluación Académica. Ha sido investigadora en el MIT Media Lab. Actualmente es consultora del proyecto Conéctate al Conocimiento de Panamá y es miembro fundadora de la Academia Internacional de Tecnología y Conocimiento AiCyT. Correo electrónico: eleonora@cariari.ucr.ac.cr
\end{abstract}

Artículo recibido: 14 de julio, 2005

Aprobado: 19 de setiembre, 2005

\footnotetext{
* Para efectos de este artículo consideramos como parte del concepto Pensum, tanto planes como programas de estudio, pues todos pueden ser diseñados de manera no lineal.
} 


\section{Introducción}

Los grandes avances de las ciencias y la revolución que han traído las nuevas tecnologías, nos colocan ante la necesidad de mirar al mundo, desde una perspectiva menos lineal y más compleja y relacional. En particular, la educación enfrenta el reto de evolucionar sus concepciones, métodos y técnicas para integrarse, armónicamente, con el rápido avance del desarrollo científico, tecnológico, ambiental y social. En este artículo, nos referiremos, muy específicamente, a la organización de pensum (englobando en este concepto tanto programas y planes de estudio) desde una perspectiva no lineal, como una alternativa que puede responder mejor a la nueva realidad. Iniciamos la presentación, haciendo una referencia muy breve a las ideas de algunos pensadores, como el sociólogo francés, Edgar Morin que propone el Pensamiento Complejo como alternativa al pensamiento lineal como un método para conocer; David Ausubel, que enfatiza en la necesidad de relacionar conceptos para que el aprendizaje sea significativo (en vez que los conceptos se presenten desconectados), y Joseph Novak que propone una herramienta conceptual particular para enlazar la teoría con la práctica: los Mapas Conceptuales. Consideramos que estas ideas son referentes conceptuales que nos permiten organizar, de una forma no lineal, nuestro entendimiento sobre el pensamiento complejo y el aprendizaje significativo. No ahondaremos en las ideas de estos autores, ampliamente reconocidos en el mundo de la educación. Partimos de sus conocidos enfoques para proponer una alternativa innovadora para el diseño de pensum no lineal. Discutimos también el potencial que tienen las tecnologías multimediales para representar el pensamiento complejo que interrelaciona en muchas dimensiones mutiplicidad de conceptos y para hacer evidente esas interrelaciones. De esta forma, las tecnologías multimediales pueden convertirse en poderosas herramientas para la organización y la representación multidimensional del pensamiento y el aprendizaje. Pero hemos dejado claro que, si bien para el diseño de pensum para programas, planes y cursos virtuales o bimodales, los multimedios son necesarios, la verdadera innovación está en el cambio del pensamiento lineal a no lineal. En el caso de diseño para pensum presencial, la herramienta puede ser o no multimedial, y por supuesto que también el interés está en el cambio de enfoque y no en la herramienta en sí. Presentamos, como alternativa, algunos ejemplos de diseño no lineal de planes de estudio de manera que las relaciones e interrelaciones entre conceptos, programas cursos y contenidos (según sea el caso) puedan mostrarse explícitamente. 


\section{Organizadores avanzados para la relacionalidad: una breve referencia de partida}

\section{II.1 Organizadores conceptuales: Pensamiento Complejo y Aprendizaje Significativo}

El sociólogo francés, Edgar Morin, uno de los principales pensadores del siglo XX, propone el Pensamiento Complejo como un método para conocer y para conocer el proceso de conocer. El pensamiento complejo es el organizador de la organización con la que representamos el mundo. Es parte de nuestros pensamientos, de nuestras ideas y nuestras teorías científicas. Tal como indica Juan Carlos Moreno (en Badilla Saxe, 2005, p. 13) lo que hoy se conoce como la teoría de la complejidad, se deriva de los desarrollos de la sistémica, de la cibernética y de la teoría de la información y la comunicación, pero se distingue de ellos porque..."la complejidad apareció como concepto sólo cuando esos desarrollos permitieron entender el papel constructivo, negantrópico, del desorden de la incertidumbre de lo aleatorio y del evento."). Morin percibe la unidad existente en la diversidad y la diversidad presente en la unidad; comprende el diálogo de las partes con el todo y del todo con las partes, así como las múltiples realidades existentes e incluye el orden y el desorden. Para Juan Carlos Moreno (en Badilla Saxe, 2005, p. 13) podemos explicarnos el pensamiento complejo desde la etimología del término "complexus", que se entiende como lo que está tejido en conjunto o lo conjuntamente entrelazado. El Pensamiento Complejo de Morin va más allá del reduccionismo mecánico, pero también trasciende el holismo: enfatiza en la necesidad de aclarar las relaciones entre las partes y el todo:

La concepción que de aquí se desprende nos sitúa de repente más allá del reduccionismo y del "holismo", apelando a un principio de inteligibilidad que integra la parte de verdad incluida en uno y otra: no debe hacer aniquilación del todo por las partes, ni de las partes por el todo. Importa, pues, aclarar las relaciones entre partes y todo, donde cada término remite al otro: "Tengo por imposible conocer las partes sin conocer el todo y también conocer el todo sin conocerlas partes", decía Pascal. (Morin, 2005, p. 150). 
Por otra parte, David Ausubel ${ }^{3}$ propone la teoría del aprendizaje significativo y plantea que los individuos, para aprender, necesitan relacionar el nuevo conocimiento a conceptos y proposiciones relevantes que conocen de antemano. Él propone que la adquisición y la retención del conocimiento (particularmente del conocimiento verbal) son el producto de un proceso activo, integrativo e interrelacional entre el contenido por aprender y las ideas relevantes que ya posee el aprendiz en sus estructuras cognitivas. A lo largo de su último libro The Acquisition and Retention of Knowledge (2000) el autor argumenta que el factor individual más importante que influye en el aprendizaje, es lo que ya sabe la persona que aprende. Agrega que el aprendizaje significativo se logra mediante procesos del conocimiento que él llama "adquisición" y "retención", los cuales no implican una actitud pasiva o acrítica de quien aprende, sino que, en un contexto más general significan, "ganar posesión" de nuevos significados. La teoría de Ausubel es concordante con el abordaje constructivista ${ }^{4}$, que propone que el sujeto construye nuevos significados y los acomoda a sus estructuras mentales. Sin embargo, Ausubel da un paso más ${ }^{5}$ al preocupase con la influencia negativa que pueden tener las relevancias ilusorias, las concepciones erróneas, los prejuicios subjetivos, la orientación motivacional hacia el aprendizaje, los estilos cognitivos y los rasgos personales, en esta construcción de significados. En su teoría del aprendizaje significativo, Ausubel aboga por la repetición multi-contextual y multi-relacional de una idea. Para él, un concepto tiene más probabilidades de ser construido y retenido si se observa o se discute o se analiza e interrelaciona en la mayoría de contextos posibles en los que resulte relevante (Ausubel 2000, p. xv). Propone que una representación global a través de organizadores avanzados, de las interrelaciones del conocimiento, puede permitir una "reconciliación integrativa" de dominios y subdominios del conocimiento por una parte y la habilidad de entender de una manera significativa las interconexiones entre los dominios y subdominios por otra. La reconciliación integrativa ocurre porque la representación del conocimiento en organizadores avanzados, puede hacer visibles y explícitas las maneras en que los conceptos aprendidos, anteriormente, están relacionados, así como el hecho de que no lo estén (en Coffey \& Cañas, 2003, pp. 275-290).

\footnotetext{
${ }^{3}$ David P. Ausubel es profesor emérito de la Universidad de la Ciudad de Nueva York.

${ }^{4}$ Representado por Jean Piaget www.piaget.org y Lev Vygotsky http://www.ibe.unesco.org/International/Publications/Thinkers/ThinkersPdf/vygotskys.PDF entre muchos otros.

${ }^{5}$ Acercándose tal vez a Vygotsky.
} 
Apoyado en la teoría del Aprendizaje Significativo, Joseph Novak (1984) propone los Mapas Conceptuales como herramienta para la representación gráfica no lineal de un dominio específico de conocimiento, mediante el conjunto de conceptos que lo conforman y sus relaciones. El mapa se construye, de tal forma, que las interrelaciones entre los conceptos se hacen evidentes. En esta graficación del conocimiento, los conceptos, se representan como nodos rotulados y las relaciones entre conceptos se manifiestan como arcos rotulados que los conectan. De esta forma, los mapas conceptuales expresan las relaciones significativas entre conceptos en forma de proposiciones o frases simplificadas: dos o más conceptos ligados por palabras para formar una unidad semántica (Cañas et al., 2000, pp. 145-158). La Figura 1 muestra un mapa conceptual sobre las aves. ${ }^{6}$

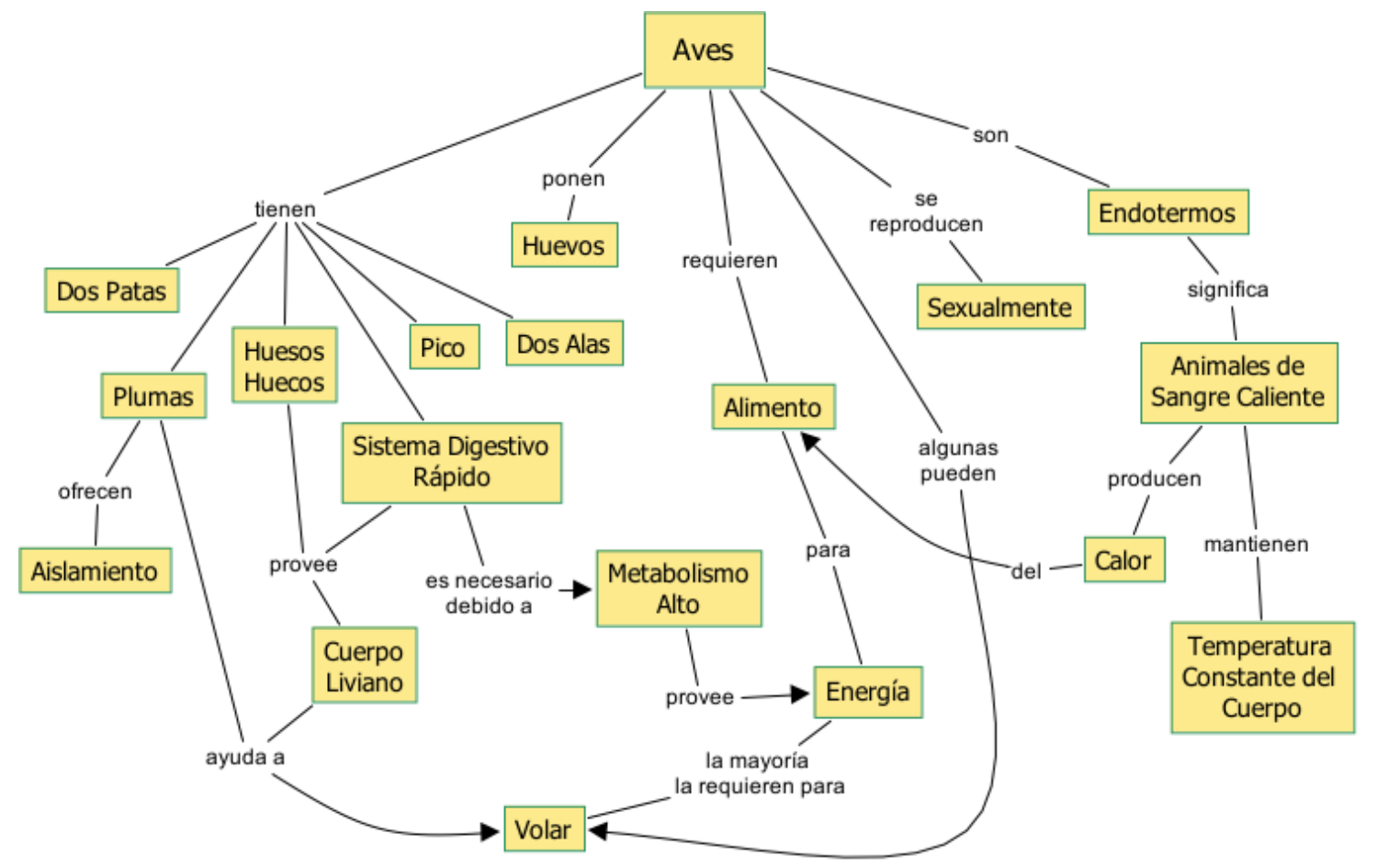

Figura $^{7} 1$

Mapa Conceptual: representación no lineal sobre las aves

\footnotetext{
${ }^{6}$ Por convención, el mapa conceptual se lee de arriba hacia abajo a menos que haya una punta de flecha en otra dirección.

${ }^{7}$ Elaborado por Fabián Cañas.
} 


\section{II.2 Organizadores tecnológicos multimediales}

El aumento en la capacidad y la velocidad de las computadoras actuales, y el alto grado de conectividad que ha logrado unir, en la red mundial, a la mayoría de las computadoras en el mundo permiten representar, de manera clara y accesible a cualquier persona, la complejidad del mundo. Un ejemplo de la evolución de las computadoras, es la red mundial Internet, que en conjunto con la World Wide Web, conocida como WWW, ha permitido que una cantidad cada vez mayor de información, esté disponible para cualquier persona, en diversos lugares y momentos, que tiene acceso a una computadora conectada a la red Internet. Esta red y la WWW tienen el potencial de interrelacionar e interconectar, de forma compleja, un vasto universo de personas, información y medios. Esa interconexión e interacción entre recursos, es lo que se conoce como hipermedios o multimedios. Sin embargo, se observa cómo la organización de la información en Internet y la WWW y las herramientas disponibles para navegar en los multimedios, no proveen un ambiente adecuado para la búsqueda de información y, mucho menos, para el aprendizaje. Al navegar por las páginas de la WWW (ver Cañas et al 2000, pp. 145-158), el usuario pocas veces tiene un modelo apropiado de la organización de estas páginas y al seleccionar las ligas o enlaces, con frecuencia desconoce el contenido de la página destino.

En general, las páginas de WWW no difieren mucho en su contenido y estructura de las páginas impresas en papel (lineales) a pesar de ser complementadas con enlaces (sin semántica). Igualmente, al utilizar páginas de WWW como base para la creación de cursos de educación a distancia, el resultado usualmente es una página de contenido o índice, con enlaces a módulos o "capítulos", organizados en páginas secuenciales, lineales. Esta imitación de la estructura de un libro de texto representa la concepción lineal de una era anterior $y$, por tanto, desaprovecha la flexibilidad que ofrecen las nuevas tecnologías que permiten representar de mejor manera, la complejidad de la actual era de las relaciones. En palabras del propio Berners-Lee (s.f., General Questions 1998, $\mathbb{1 1 1}$ ), creador de la WWW:

Hay muchas partes del sueño original que aún no han sido implementadas. Por ejemplo, pocas personas tienen una herramienta fácil e intuitiva para poner sus pensamientos en hipertexto. $Y$ muchas de las razones y significados de los enlaces de la Web se pierden. Pero esto puede y creo que va a cambiar. 
Con esta cita, Berners-Lee nos dice que, a pesar del enorme potencial de los hipermedios y en particular de su sueño al crear la WWW, al no aclararse la relación entre las páginas, no se encuentra la semántica y, con ello, no se entiende el significado. Por ejemplo (Cañas, 2005 (b)), si en una página Web tenemos la siguiente oración: "La Universidad de Costa Rica

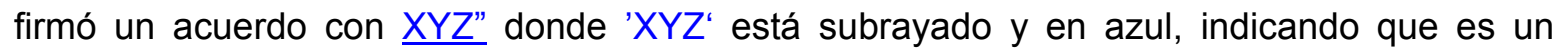

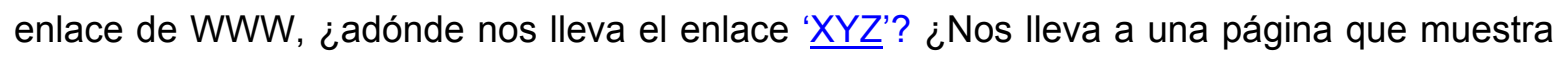
el acuerdo? O ¿nos lleva a la página www.xyz.org? Si ambos, Universidad de Costa Rica y XYZ están subrayados, ¿cada enlace lleva a la página WWW de cada organización? $\mathrm{O}$ ¿tal vez a una descripción de la contribución/participación de cada organización en el acuerdo? No hay forma de saberlo, a menos de que sigamos cada enlace o se haga explícito en el texto anterior o posterior al enlace. O sea, el enlace en sí, no aclara la relación entre las páginas. A esto se le llama, en algunos círculos: "enlaces sin semántica", es decir, que no tienen significado.

En la siguiente dirección en la WWW, se puede observar un ejemplo ilustrativo de cómo se utilizan los hipermedios, la internet y la WWW, para reproducir esquemas mentales lineales, con "enlaces sin sentido":

\section{http://www.aulafacil.com/CursoContabilidad/clase1-1.htm ${ }^{8}$}

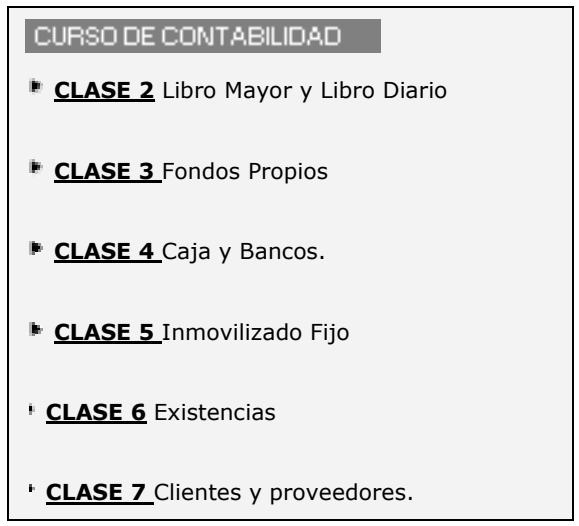

En este caso, se trata de un curso de Contabilidad, donde las clases, los contenidos y los conceptos se listan de una manera lineal, sin que se pueda entender cuál es la relación entre ellos. El enorme potencial de relacionalidad de los hipermedios y la WWW es

${ }^{8}$ Consultada el 10 de julio de 2005. Este listado de contenidos, que se copia textualmente, se encuentra en la columna de la izquierda del sitio WWW mencionado. 
desaprovechado en estas situaciones y, a pesar de la presencia de "enlaces" entre palabras, no existe una verdadera interrelacionalidad entre conceptos, es decir, no son semánticos, no tienen sentido. Más bien, reproducen esquemas de pensamiento y de representación del conocimiento lineal, ampliamente superados por los aportes de las ciencias, el desarrollo de las teorías del pensamiento y el aprendizaje, así como las posibilidades relacionales de las tecnologías de la información y la comunicación.

Por otra parte, cuando anteriormente se cita a Berners-Lee sobre la falta de semántica en el WWW, él se refería a la "Web Semántica" (“Semantic Web", la nueva versión de WWW) donde los enlaces se especifican de forma tal que una máquina pueda entender esa relación (las relaciones se basan en lenguajes formales y ontologías). En nuestro caso, donde nos interesa el aprendizaje por parte de humanos, no de máquinas, el interés no se centra en que la computadora "entienda" los enlaces, sino en que sean entendibles para las personas (Cañas, 2005 (b), en comunicación electrónica conocida como "chat").

Es posible y necesario aprovechar el potencial de organización y representación no lineal del conocimiento que ofrecen tanto los Mapas Conceptuales, como los hipermedios y la WWW, para trascender los esquemas en evolución del pensamiento lineal al pensamiento relacional. CmapTools ${ }^{9}$ es una herramienta de software, diseñada y creada especialmente con este fin que permite utilizar los Mapas Conceptuales como una interfaz elegante y fácil de comprender para navegar en un sistema de multimedia (Cañas et al 2005, pp. 205-219).

Un ejemplo de esta interfaz es la representada por la página principal del Centro de Evaluación Académica (CEA), de la Universidad de Costa Rica (UCR). En ella, se establecen las relaciones entre las dependencias que lo conforman y entre esas dependencias con las diversas unidades en el interior de la universidad. Otro ejemplo que ilustra la navegación interrelacional es la figura 2 que muestra un modelo construido por un niño que utiliza mapas para organizar imágenes, vídeo, y texto. Las relaciones de generalización y especialización entre los conceptos conllevan a una organización jerárquica de mapas conceptuales. Al posibilitar que el modelo sea accesible en Internet, éste se vuelve navegable por otras personas, ya sean estudiantes, docentes o usuarios de la red en general.

\footnotetext{
${ }^{9}$ El CmapTools es un software diseñado en el Institute for Human and Machine Cognition (IHMC) para construir, navegar y criticar modelos de conocimientos representados como mapas conceptuales. Mayor información disponible en: http://cmap.inmc.us/
} 


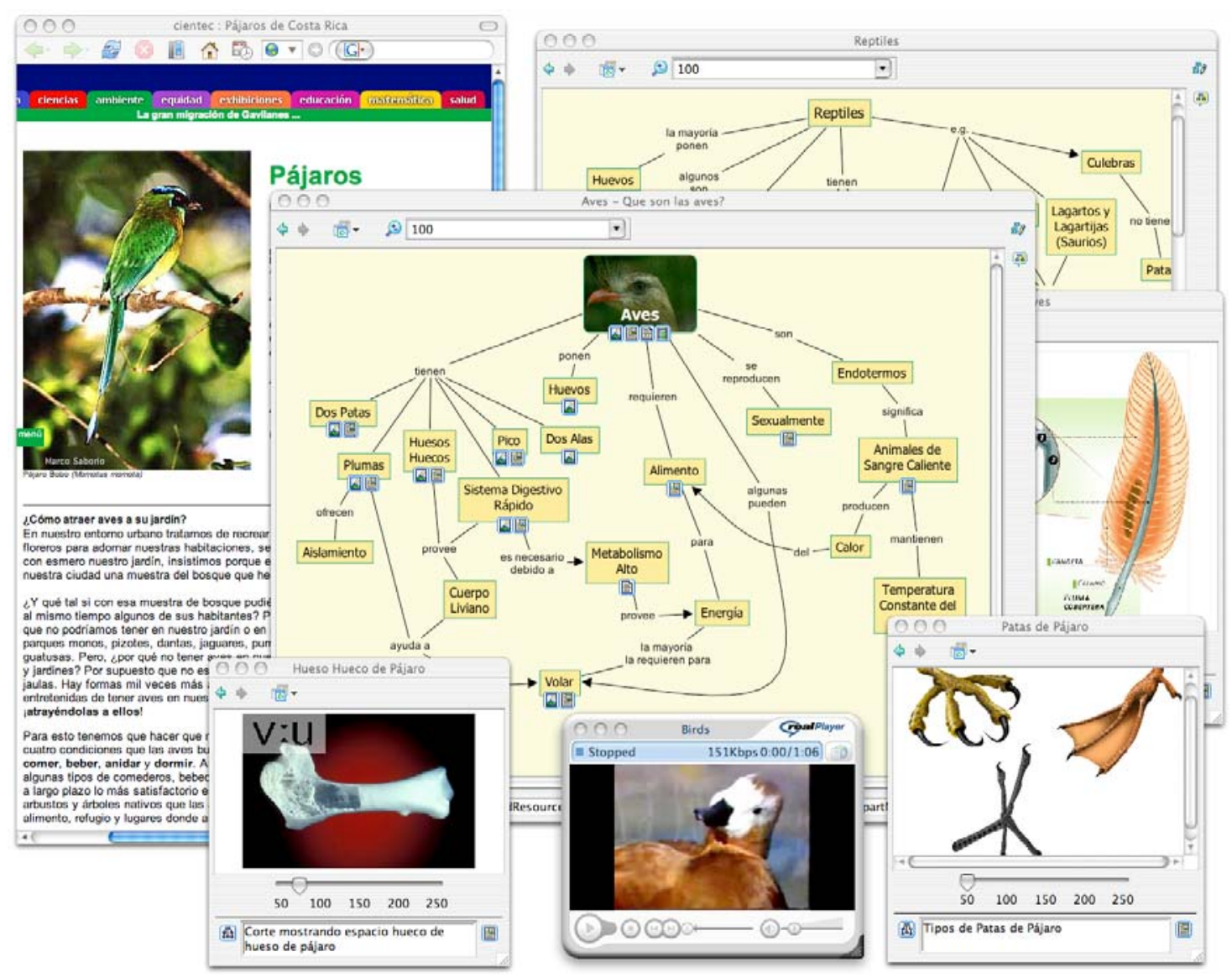

Figura 2

Mapas Conceptuales como medio para navegar por un ambiente no lineal y multi-relacional y multi-medial sobre las aves, basado en el mapa de la Figura 1.

En cada nodo o concepto del mapa, el usuario dispone de un menú de íconos que fueron agregados por la persona que construyó el mapa. Estos corresponden a diversos medios (texto, imágenes, vídeo, otros mapas conceptuales, etc.) relacionados al tema del nodo (concepto) seleccionado. Al seleccionar un ícono, el sistema despliega información de otros recursos que contienen información referente al concepto. Por ejemplo, el concepto "Aves" en la Figura 2 contiene cuatro íconos, como se observa en la Figura 3. 


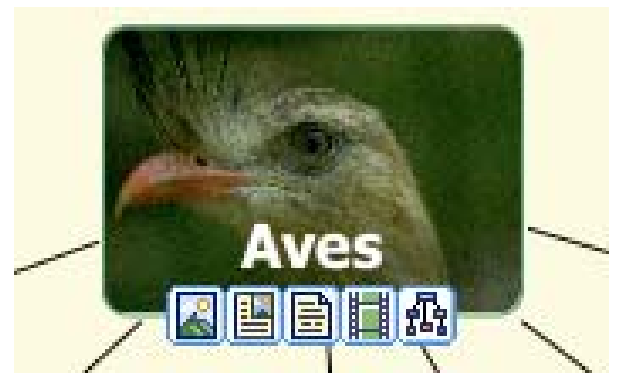

Figura 3

Concepto raíz del mapa conceptual de la Figura 2 e íconos que permiten navegar a diferentes tipos de recursos asociados con el concepto y el mapa.

- El ícono de la extrema izquierda representa una imagen que, al ser seleccionada con el ratón, despliega una lista descriptiva de imágenes de aves. El usuario puede seleccionar aquella que desea desplegar.

- El segundo ícono muestra una lista de textos, con imágenes, explicativas sobre aves.

- El tercero representa un texto y contiene informaciones sobre aves.

- El cuarto ícono de este concepto representa un video. Al seleccionarlo se despliega una lista de videos sobre aves que están disponibles.

- El ícono de la extrema derecha representa un mapa conceptual que permite enlazar mapas conceptuales relacionados. Al seleccionar este ícono, se despliega una lista de mapas conceptuales relacionados con las aves (no necesariamente mapas sobre aves). En la Figura 2, el mapa conceptual sobre reptiles que se encuentra parcialmente tapado, tiene un concepto "aves" que enlaza ese mapa con el de aves.

En cualquier caso, la lista desplegada, al seleccionar alguno de los íconos, indicará claramente, si al navegar en el mapa, ocurrirá un cambio de contexto. Por ejemplo, si bajo el concepto "volar" hubiera un enlace a un mapa sobre "vuelo", pero este mapa se refiere al vuelo dentro del contexto de aviación, al encontrarse el mapa sobre vuelo en otro modelo de conocimiento, el enlace automáticamente indicaría que el contexto es diferente y se desplegaría como "aviación: vuelo", permitiendo al usuario decidir, si le interesa seguir el enlace o no.

Los íconos aparecen en diversas combinaciones, dependiendo de la información disponible sobre el concepto dado. El problema de navegación -perdidos en el hiperespacio- común en los sistemas de multimedia y persistente en la navegación de la WWW, se resuelve 
fácilmente en la navegación con Mapas Conceptuales. Todas las ligas tienen semántica, ya sea explícita en el mapa, o por contexto, al navegar entre mapas u otros medios. El usuario sabe siempre hacia dónde va al seguir una liga, en contraste con otras herramientas de navegación, especialmente en la WWW (ver Cañas et al, 2000, pp. 145-158). Adicionalmente, los íconos indican el tipo de información enlazado (imagen, texto, texto e imagen, video, mapa conceptual, etc.) sin importar o hacer referencia al formato en que se encuentra el medio (e.g. jpg, gif, avi, pdf, etc.).

\section{Del Pensum lineal al Pensum no lineal}

Los Pensum (programas o planes de estudio) que hemos diseñado tradicionalmente en educación, en el contexto de una visión curricular determinada o un enfoque pedagógico particular, se derivan de los esquemas de pensamiento lineales. Generalmente, salvando contadas excepciones, se trata de listados de cursos inconexos entre sí (organizados, por lo demás, en una línea de tiempo secuencial) que, a su vez incluyen listados de contenidos independientes, que se conforman listas de conceptos aislados. Como ejemplo ilustrativo, podemos revisar rápidamente algunos planes de estudio de la Universidad de Costa Rica, los cuales se encuentran en el sitio de su Centro de Evaluación Académica CEA:

\section{http://www.cea.ucr.ac.cr/diea/estructuras/}

No es arriesgado afirmar que, en un altísimo porcentaje, los planes de estudio de universidades nacionales e internacionales, así como del sistema educativo nacional responden a este modelo lineal de diseño. En la página Web del Ministerio de Educación de Costa Rica, podemos revisar también los planes de estudio del sistema educativo nacional, actualizados al 2005 http://www.mep.go.cr/PlanesEstudios2005.html para corroborar que se trata de listas de contenidos.

Para desempeñarse de manera satisfactoria y exitosa en el nivel personal y profesional en la Era de las Relaciones, las jóvenes generaciones, más que repetir conceptos desconectados, deben tomar decisiones complejas basadas en el análisis de grandes cantidades de datos e información. En el mundo actual, es necesario realizar operaciones cognitivas complejas que implican multiplicidad de relaciones e involucran entender y hacer generalizaciones sobre una estructura cognitiva básica (ver Coffey \& Cañas, 2003, pp. 275-290). Sin embargo, es una realidad que los planes de estudio del sistema educativo, desde los niveles de educación básica hasta universitaria, están diseñados para promover un pensamiento lineal, rígido y no relacional, como consecuencia de un diseño lineal, rígido, fragmentado y 
descontextualizado. Claro, no se trata de reducir lo educativo a este tema, pues se sabe que hay muchas otras variables que deben ser analizadas desde una perspectiva crítica.

Resulta evidente, entonces, la necesidad de buscar formas alternativas de diseñar y organizar los Pensum, de manera que respondan a las demandas de la época y promuevan el pensamiento complejo y el aprendizaje significativo, y se puedan aprovechar las posibilidades que actualmente ofrecen las tecnologías multimediales y multi-relacionales.

\section{- Mapas Conceptuales para el diseño de pensum no lineal}

Una opción para diseñar planes de estudio más flexibles, es utilizar la herramienta de Mapas Conceptuales. Como se indicó anteriormente, es una herramienta que permite la organización no lineal del conocimiento, así como explicitar las interrelaciones entre conceptos, cursos, y contenidos.

Actualmente se observa una tendencia a utilizar mapas conceptuales y herramientas multimediales para el diseño de pensum no lineales (ver Novak \& Symington, 1982; Moen \& Boersma, 1997; Allen 1993), en disciplinas diversas como el currículo para la formación de médicos (ver Edmondson 1993), o para el aprendizaje de las ciencias (ver Starr \& Krajcik 1990; Williams 1997; Ferry et al, 1998).

En las Figuras 4 y $5^{10}$ hay dos ejemplos de cursos de Toma de Decisiones Gerenciales y Psicología, organizados como un mapa conceptual.

${ }^{10}$ Tomadas de Cañas, 2005 (a). Ver también Arguea \& Cañas (1998). 


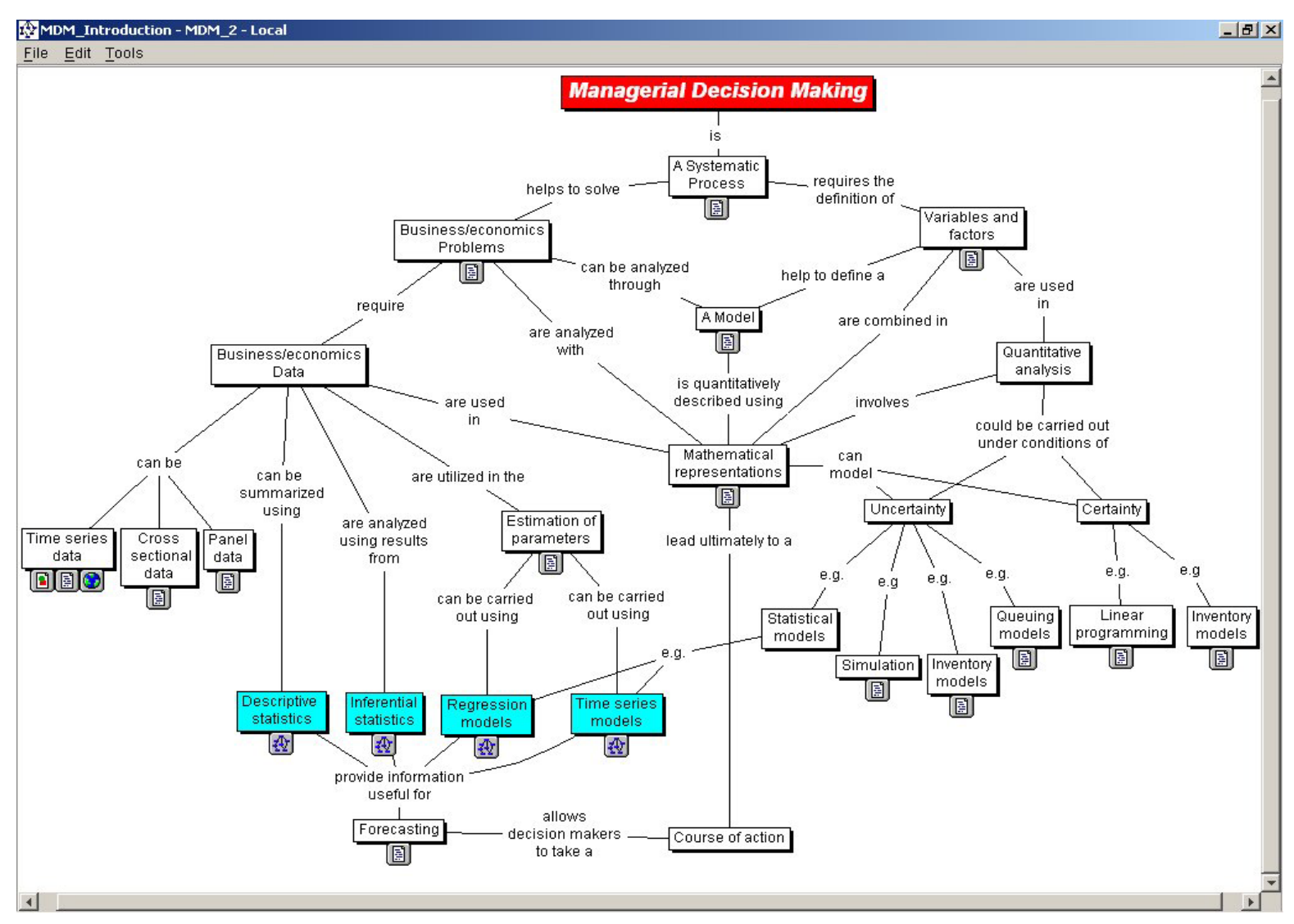

Figura 4

Diseño no lineal de un curso sobre Toma de Decisiones Gerenciales utilizando mapas conceptuales 


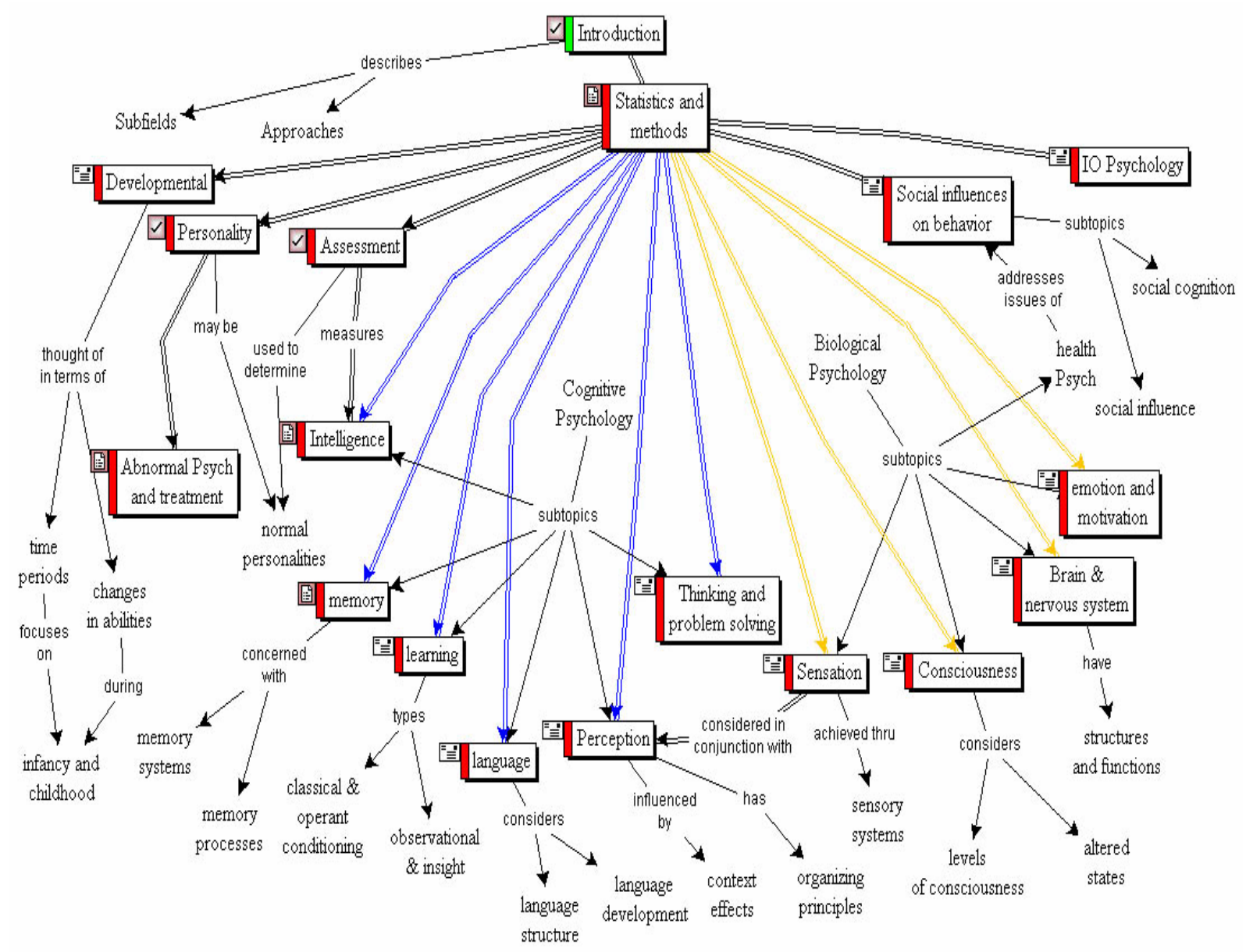

Figura 5

Diseño no lineal de un curso de Psicología utilizando mapas conceptuales

En el caso de la Figura 4, en el diseñó se utilizó el software CmapTools. En el caso de la Figura 5, el diseño fue realizado sobreponiendo una secuencia al mapa conceptual, mediante una extensión a CmapTools conocida como LEO, un organizador de ambientes de aprendizaje en red, aún no disponible, pues se encuentra en la etapa de investigación (ver Coffey \& Cañas, 2003, pp. 275-290). El mapa conceptual se somete al análisis con el programa LEO que, a su vez, genera las líneas amarillas y azules que se observan y que pueden representar requisitos, co-requisitos o recomendaciones del o la profesora guía para el estudiante. En el caso del diseño de planes, carreras o cursos totalmente presenciales, el uso de herramientas multimediales es altamente deseable, aunque no imprescindible. El 
argumento de fondo es que hay que evolucionar de un pensamiento lineal a un pensamiento relacional y complejo.

En el caso de planes, carreras y cursos bimodales o virtuales, por el contrario, el uso de herramientas multimediales es imprescindible, pero no suficiente. Ya hemos visto en ejemplos presentados anteriormente, cómo las más modernas tecnologías son utilizadas para reproducir esquemas mentales superados. En todos los casos, el primer paso es un cambio de visión y de perspectiva. El siguiente paso es buscar, encontrar y utilizar las mejores herramientas de las que se pueda disponer.

\section{- Módulos creados por expertos}

Otra alternativa, (que complementa y enriquece la anterior), para el diseño de planes y cursos no lineales, es la creación de módulos sobre un tema general (ver Cañas, 1999, consultada en agosto 2005). Por un módulo entendemos aquí una parte prediseñada que se puede integrar y relacionar de distintas maneras con el todo (plan o curso según el caso). El módulo, que sería diseñado por un experto en el tema, para integrarse (dependiendo del caso) al plan o a un curso, debería estar conceptualizado y desarrollado en forma armónica con la concepción integral del pensum o curso; es decir, de forma no lineal, y utilizando herramientas tecnológicas compatibles. La flexibilidad de creación de contenidos en el nivel de módulos, permite buscar en el diseño de un pensum o la preparación de un curso, las mejores versiones de cada uno de los módulos, según las necesidades del plan o del curso. Es importante entonces encontrar a la persona más calificada -- al "experto" o la "experta" en la materia -- para la creación de cada uno de estos módulos. Al experto o experta la caracteriza su experiencia, habilidad, o pericia, en un tema particular. Los mapas han sido usados con éxito en el desarrollo de sistemas expertos (Ver Cañas 1999, consultada agosto 2005) y, específicamente, en la representación de modelos del conocimiento de expertos.

Por supuesto que debe haber pre-requisitos de conocimiento que el estudiante deberá cumplir para trabajar en un módulo de una carrera o en el de un curso; pero hasta donde sea posible, no deben depender de una secuencia fija de módulos anteriores. De esta manera, el o la profesora puede reorganizar el orden en que se cubren los módulos (la variación puede inclusive ser para cada estudiante), o utilizar solamente algunos de ellos. Los módulos deben poder hacer referencias a otros módulos.

La Figura 6 es una representación del modelo de conocimiento sobre Marte (que puede ser utilizado para incluir en un pensum no linea), construido por un experto en el tema (Briggs et al, 2004). En este caso, el director del Centro de Exploración de Marte de la NASA, utilizó 
CmapTools para el desarrollo de una colección de docenas de mapas conceptuales y miles de recursos sobre Marte que ilustran cómo un experto puede crear módulos sobre su tema de especialidad, sin importar la secuencia que seguiría al utilizar ese contenido en cursos.

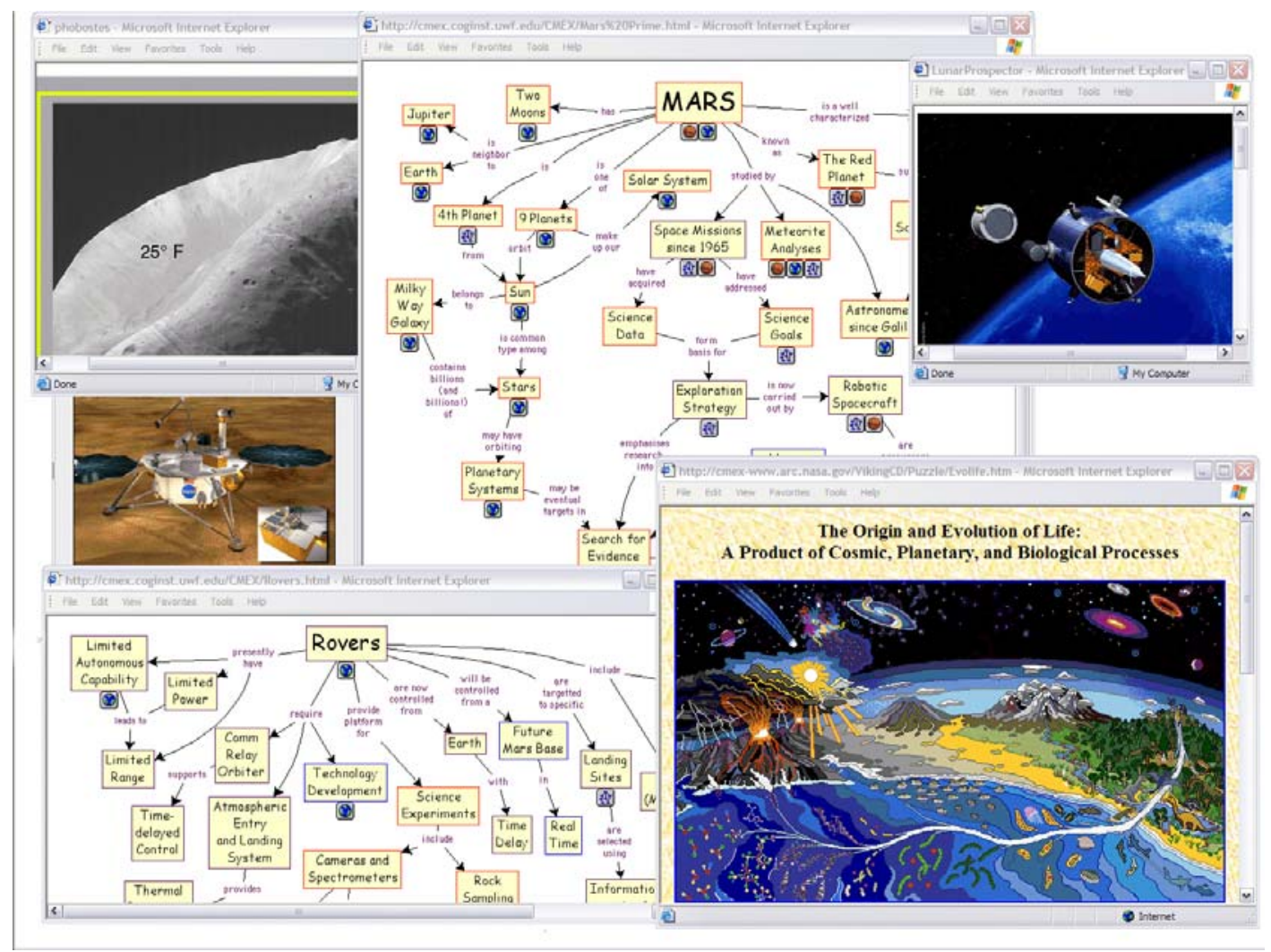

Figura 6

Mapa Conceptual sobre Marte elaborado por un experto, el director del Centro de Exploración de Marte de la NASA. Forma parte de una conjunto de docenas de mapas y miles de recursos sobre Marte (tomado de Briggs et al (2004).

De nuevo, el diseño modular de planes, cursos y contenidos de planes o cursos que se realizarán de manera exclusivamente presencial, puede verse definitivamente enriquecido con el uso de herramientas multimediales; pero de no tener acceso a éstas, el requisito más importante es el cambio de pensamiento lineal a complejo.

$\mathrm{Si}$ los planes, cursos y contenidos son bimodales o totalmente virtuales, el uso de herramientas multimediales es necesario, aunque también se insiste en que la condición fundamental es que se comprenda la importancia de la relacionalidad y se abandone la linealidad. En este caso, es importante tener presente que los módulos deben ser, por llamarlo así, "tecnológicamente compatibles." Sería una verdadera dificultad, si para cada Volumen 5, Número Especial, Año 2005, ISSN 1409-4703 16 
módulo necesitáramos software diferente, con interfaces distintas, cada una con su curva de aprendizaje. Por supuesto que deben existir pre-requisitos de conocimiento que el estudiante deberá cumplir para trabajar en un módulo; pero hasta donde sea posible, no deben depender de una secuencia fija de módulos anteriores. Los módulos deben contar con la posibilidad de hacer referencias a otros módulos, lo cual significa también un alto grado de compatibilidad. Finalmente, debe existir un programa informático que permita la integración de todos los módulos (ver Cañas, 1999, consultada agosto 2005).

\section{Conclusiones}

Las demandas del mundo actual presentan el enorme reto de enfrentar, conocer y entender nuevos conocimientos y tecnologías. Pero más allá de eso, obligan a un cambio en la perspectiva lineal que tenemos del mundo, para aceptar la relacionalidad de la realidad. Esto segundo, posiblemente, es más difícil que lo primero.

Esta situación tiene implicaciones directas en la organización y representación del conocimiento, así como en el aprendizaje, sobre todo de las nuevas generaciones. Más allá de la simple repetición de conceptos inconexos, la época actual exige de las personas y profesionales, capacidad para la toma de decisiones complejas, basadas en el análisis de grandes cantidades de datos e información, así como realizar operaciones cognitivas complejas que implican multiplicidad de relaciones, así como entendimiento y establecimiento de generalizaciones sobre una estructura cognitiva básica.

Evidentemente, los sistemas educativos, por multiplicidad de razones, no están promoviendo ese pensamiento complejo en los y las estudiantes. Una de esas razones, que interesa particularmente en este artículo, es preguntarse: ¿por qué los planes de estudio, los cursos y los contenidos están organizados de forma lineal, rígida y a partir de listados de conceptos desconectados entre sí?

En el complejo contexto de la educación, es importante prestar atención a cambiar, para comenzar el diseño mismo de los planes, cursos y contenidos. Es necesario pasar de las listas inconexas, a mapas interrelacionados que den significado y promuevan el Pensamiento Complejo.

Los Mapas Conceptuales son una de las alternativas con las que contamos para trascender del diseño lineal, al diseño multi-relacional en la organización y representación del conocimiento. Un enfoque de creación de módulos por expertos podría complementar el diseño del plan de estudios con mapas conceptuales. En el caso de diseños para planes y cursos totalmente presenciales, el uso de herramientas multimediales es altamente 
deseable, aunque no imprescindible. En el caso de la educación bimodal o virtual, si bien el uso de herramientas multimediales en su diseño es imprescindible, no es suficiente. El primer paso debe ser un cambio de visión y de perspectiva. En segundo lugar queda buscar, encontrar y utilizar las mejores herramientas de las que se puedan disponer para el diseño no lineal de un pensum o plan de estudios.

\section{Referencias}

Allen, B. S. et al. (1993). Computer-Based Mapping for Curriculum Development. In Proceedings of Selected Research and Development Presentations at the Convention of the Association for Educational Communications and Technology Sponsored by the Research and Theory Division. New Orleans, Louisiana: AECTC Press.

Arguea, N. \& A. J. Cañas. (1998). Mapas Conceptuales como Herramienta en Estadística Aplicada: Una Propuesta para un Curso a Distancia. Memoria del IX Congreso Internacional sobre Tecnología y Educación a Distancia. San José, Costa Rica: EUNED.

Ausubel, D. P. (2000). The Acquisition and Retention of Knowledge: A Cognitive View. Dordrecht, Londres: Kluwer Academic Publishers.

Badilla Saxe, E. (2005 en imprenta). Educación: Relaciones y Conexiones, Alajuela: Editorial CUNA.

Badilla Saxe, E. (2003) Herramientas Mentales y Digitales para Desmitificar y Democratizar la Información. Recuperado en julio de 2005, en: http://www.cientec.or.cr/comunicacion/ponencias/EleonoraBadilla.pdf

Badilla Saxe, E. (1997). Educarse en el umbral del milenio. En Serie Concurrencia, Documentos Ocasionales, N. 15. San José: PROMECE, MEP.

Berners-Lee, Tim. Tim Berners-Lee's Webpage, en http://www.w3.org/People/BernersLee/FAQ, consultada en Julio 2005

Briggs, G., D. A. Shamma, A. J. Cañas, R. Carff, J. Scargle, J. D Novak. (2004). Concept Maps Applied to Mars Exploration Public Outreach. In A. J. Cañas, J. D. Novak, \& F. M. González (Eds.). Concept Maps: Theory, Methodology, Technology, Proceedings of the First International Conference on Concept Mapping. Pamplona, Spain: Editorial Universidad Pública de Navarra.

Cañas, A. J. (2005 a). Comunicación personal. Presentación sobre Pensum no lineal el 6 de mayo, en la Sala del Consejo Universitario, Universidad de Costa Rica.

Cañas, A. J. (2005 b). Sobre Relacionalidad. Foro Digital grupo 10. San José, Costa Rica: Doctorado en Educación, Universidad La Salle. 
Cañas, A. J. (1999) Algunas Ideas sobre la Educación y las Herramientas Computacionales Necesarias para Apoyar su Implementación. Revista RED: Educación y Formación Profesional a Distancia, Ministry of Education, España. (Disponible en http://www.ihmc.us/users/acanas/Publications/IdeasEnEducacion/ACanas\%20ldeas\% 20Educacion.htm).

Cañas, A. J. et al, (2000) Herramientas Para Construir y Compartir Modelos de Conocimiento Basados en Mapas Conceptuales. Revista de Informática Educativa, Vol. 13, N² 2000, pp. 145-158. (http://lidie.uniandes.edu.co/revista)

Cañas, A. J., G. Hill, R. Carff, N. Suri, J. Lott, G. Gómez, T. C. Eskridge, M. Arroyo, R. Carvajal. (2004). CmapTools: A Knowledge Modeling and Sharing Environment, In A. J. Cañas, J. D. Novak, \& F. M. González (Eds.), Concept Maps: Theory, Methodology, Technology, Proceedings of the First International Conference on Concept Mapping. Pamplona, Spain: Editorial Universidad Pública de Navarra.

Cañas, A. J., R. Carff, G. Hill, M. Carvalho, M. Arguedas, T. C. Eskridge, J. Lott, R. Carvajal (2005). Concept Maps: Integrating Knowledge and Information Visualization. In S.-O. Tergan \& T. Keller, Heidelberg Eds.) Knowledge and Information Visualization: Searching for Synergies (pp. 205-219). New York: Springer Lecture Notes in Computer Science,

Coffey, J. \& Cañas, A. J. (2003). LEO: A Learning Environment Organizer to Support Computer-Mediated Instruction. Journal for Educational Technology Systems 31(3), pp. 275-290.

Edmondson, K. M. (1993). Concept mapping for the development of medical curricula. Paper presented at the Annual Conference of the American Educational Research Association, Atlanta, GA. (Eric Document Reproduction Services No. ED 360 322).

Ferry, B., Hedberg, J., \& Harper, B. (1998). How Do Preservice Teachers Use Concept Maps To Organize Their Curriculum Content Knowledge? Journal of Interactive Learning Research. 9(1): p. 83-104.

Moen, E.M.J.C. \& Boersma, K.T. (1997). The Significance of Concept Mapping for Education and Curriculum Development. Journal of Interactive Learning Research. (3-4): p. 487-502.

Morin, Edgar. (2005). La Organización: del objeto al sistema. En Antología sobre Pensamiento Complejo. San Pedro, Costa Rica: Centro de Evaluación Académica, UCR.

Novak, J. D. \& Gowi, D. B.. (1984). Learning How to Learn. New York: Cambridge University Press.

Novak, J.D. \& Symington, D. (1982). Concept mapping for curriculum development. V.I.E.R. (The Victorian Institute of Educational Research). 
Starr, M. L., \& Krajcik, J. S. (1990). Concept Maps as a heuristic for science curriculum develomment: Towards improvement in process and product. Journal of Research in Science Teaching, 27(10), 987-1000.

Williams, M.A. (1997). Integrating Concept Mapping into Science Curriculum and Instructional Practice: Teacher Experiences, Observations, and Recommendations for Future Projects. Journal of Interactive Learning Research, 8(3-4): p. 457-485. 